\title{
Structural damage detection using principal component analysis and damage indices
}

Journal of Intelligent Material Systems and Structures

I-I6

(c) The Author(s) 2015

Reprints and permissions:

sagepub.co.uk/journalsPermissions.nav DOI: I0.1 I77/I045389X|4566520 jim.sagepub.com

\author{
Diego A Tibaduiza', Luis E Mujica ${ }^{2}$, José Rodellar ${ }^{2}$ and Alfredo Güemes ${ }^{3}$
}

\begin{abstract}
One of the most important tasks in structural health monitoring corresponds to damage detection. In this task, the existence of damage should be determined. In the literature, several potentially useful techniques for damage detection can be found, and their applicability to a particular situation depends on the size of the critical damages that are admissible in the structure. Almost all of these techniques follow the same general procedure: the structure is excited using actuators, and the dynamical response is sensed at different locations throughout the structure. Any damage will change this vibrational response. The state of the structure is diagnosed by means of the processing of these data. Several studies have shown that the detection of changes in a structure depends on the distance from the damage to the actuator as well as the configuration of the sensor network. In this article, the authors considered the advantage of using an active piezoelectric system, where the lead zirconate titanate transducers are used as actuator and sensors in different actuation phases. In each actuation phase of the diagnosis procedure, one lead zirconate titanate transducer is used as actuator (a known electrical signal is applied), and the others are used as sensors (collecting the wave propagated through the structure at different points). An initial baseline model for undamaged structure is built applying principal component analysis to the data collected by several experiments and after the current structure (damaged or not) is subjected to the same experiments, and the collected data are projected into the principal component analysis models. Two of these projections and four damage indices ( $T^{2}$-statistic, $Q$-statistic, combined index, and $I^{2}$ index) by each actuation phase are used to determine the presence of damages and to distinguish between them. These indices are calculated based on the analysis of the residual data matrix to represent the variability of the data projected within the residual subspace and the new space of the principal components. To validate the approach, data from two aeronautical structures-an aircraft skin panel and an aircraft turbine blade—are used.
\end{abstract}

\section{Keywords}

principal component analysis, damage detection, damage indices, active piezoelectric system

\section{Introduction}

Structural health monitoring (SHM) is an area in which the main objective is the verification of the state or the health of structures in order to ensure proper performance and maintenance cost savings using nondestructive tests, sensors permanently attached to the structure, and computational algorithms. Different benefits are derived from the implementation of SHM, some of which are knowledge about of the behavior of the structure under different loads and different environmental changes, knowledge of the current state in order to verify the integrity of the structure and to determine whether a structure can work properly or whether it needs maintenance or replacement, and therefore, maintenance cost saving. The paradigm of damage identification (comparison between the data collected from the structure without damages and the current structure in order to determine whether there are any changes) can be tackled as a pattern

\footnotetext{
'MEM (Research Group on Monitoring-Electronics and Modelling), Faculty of Electronics Engineering, Universidad Santo Tomás-Colombia, Bogotá, Colombia

${ }^{2}$ CoDAlab, Departament de Matemàtica Aplicada III, Escola Universitària d'Enginyeria Tècnica Industrial de Barcelona (EUETIB), Universitat Politècnica de Catalunya-BarcelonaTech (UPC), Barcelona, Spain

${ }^{3}$ Center of Composites Materials and Smart Structures, ETSIA,

Universidad Politécnica de Madrid, Madrid, Spain
}

\section{Corresponding author:}

Diego A Tibaduiza, MEM (Research Group on Monitoring-Electronics and Modelling), Faculty of Electronics Engineering, Universidad Santo TomásColombia, Cra 9 No. 5 I-II Bogotá, Colombia.

Email: diegotibaduiza@usantotomas.edu.co 
recognition application (Farrar et al., 2004); in this sense, some statistical techniques such as principal component analysis (PCA) are very useful for pattern recognition and data processing.

PCA has been extensively applied to measure structural dynamic response signals with the purpose of dimensionality reduction studies (Manson et al., 2001; Mujica et al., 2008) to distinguish between changes due to environmental and structural damage (Manson, 2002) and for sensor validation (Kerschen et al., 2005), among others. The authors have been recently using PCA in SHM applications, specifically for damage classification using self-organizing maps (Tibaduiza et al., 2012, 2013) and damage localization tasks (Tibaduiza et al., 2011). In these works, data from the structure are collected by a piezoelectric system in several actuation phases. The recorded data are pre-processed and organized to calculate a PCA model and some damage indices by each actuation phase. In the localization tasks, some contribution methods are used to calculate the contribution of each sensor to each index and to determine the location of the damage. For classification task, the results obtained by each actuation phase are used in conjunction with a Self-Organizing Map to classify different states of the structure. This article uses the same methodology to inspect the structures - this means an active piezoelectric system which involves the use of piezoelectric transducers that are attached to the surface of the structure in order to produce Lamb waves and collect the signals propagated through the structure at different locations and working in several actuation phases. As pattern recognition technique, PCA is used to perform the analysis - by building a baseline model of the structure without damage - and, subsequently, to compare the data from the current state of the structure (damaged and undamaged). This comparison is performed using the first two projections into the PCA model (score 1 and score 2) and four damage indices also based on PCA $\left(T^{2}, Q, I^{2}, \Phi\right)$ to determine whether there are differences between the signals collected when the structure is known as healthy and the structure is in an unknown state. The methodology is tested using data from an aircraft turbine blade and an aircraft skin panel. The proposed methodology presents new contributions because it shows that scores are not the best solution for damage detection when a few principal components (PCs) with low retained variance are used and introduces the use of the indices $\left(I^{2}, \Phi\right)$ as possible solution for the damage detection problem by considering the use of data from a piezoelectric system working in several actuation phases. In addition, it presents new ways to interpret the results from the data-driven analysis, which is applied to experimental setup by considering these statistical damage indices and a comparison of the results from the different actuation phases. From the knowledge of the authors, there is no similar study with similar results involving the use of all these indices.

This article is organized in six sections starting with this introduction. In section "Theoretical background," a brief theoretical background about PCA and damage indices is introduced. Afterward, section "Damage detection methodology" presents the damage detection methodology developed. Sections "Experimental setup" and "Experimental results" present the experimental setup and the experimental results. Finally, the conclusions are discussed in section "Conclusion."

\section{Theoretical background}

\section{PCA}

PCA is a classical technique of multivariable analysis, and its theory is well documented in any textbook (e.g. Jolliffe, 2002). To apply PCA, it is necessary to arrange the collected data in a matrix $\boldsymbol{X}$ which is previously normalized. This $n \times m$ matrix contains information from $m$ sensors and $n$ experimental trials (Mujica et al., 2011). This matrix $\boldsymbol{X}$ is used to calculate the covariance matrix $C_{x}$ as follows

$$
C_{x} \equiv \frac{1}{n-1} \boldsymbol{X}^{\mathrm{T}} \boldsymbol{X}
$$

It is a square symmetric $m \times m$ matrix that measures the degree of linear relationship within the dataset between all possible pairs of variables (sensors). The subspaces in PCA are defined by the eigenvectors and eigenvalues of the covariance matrix as follows

$$
C_{x} \boldsymbol{P}=\boldsymbol{P} \Lambda
$$

where the eigenvectors of $C_{x}$ are the columns of $\tilde{\boldsymbol{P}}$, and the eigenvalues are the diagonal terms of $\Lambda$ (the offdiagonal terms are zero). The PCs correspond to the columns of matrix $\tilde{\boldsymbol{P}}$ which are sorted according to the eigenvalues by descending order. In this way, the new matrix $\boldsymbol{P}(\tilde{\boldsymbol{P}}$ sorted and reduced $)$ can be called as PCA model. Geometrically, the transformed data matrix $\boldsymbol{T}$ (score matrix) represents the projection of the original data over the direction of the PCs $\boldsymbol{P}$

$$
T=X P
$$

\section{Damage detection indices based on PCA}

PCA is a well-known statistical technique that has been used as a pattern recognition technique by several years with excellent result. Its use allows obtaining patterns that often underlie from the data by calculating the PCs and re-expressing the information in a new space. In this article, PCA allows defining patterns from the 
structure when it is known as healthy to define the baseline by each actuator phase. A comparison between dynamical signals of the structure to analyze and a baseline (pattern) allows determining whether some changes exist and, besides, whether these changes can be considered as a damage or not. Transforming or projecting data from different states of the structure by using PCA would permit an easy comparison between them. Sometimes, these projections are not enough, and it is necessary for the use of some statistical measurements that can be considered as damage indices.

There are several kinds of indices that can give information about the accuracy of the model and/or the adjustment of each experiment to the model. Two wellknown indices are commonly used to this aim: the $Q$ statistic (or square prediction error [SPE] index) and the Hotelling's $T^{2}$-statistic ( $D$ index) (Alcalá and Qin, 2009; Mujica et al., 2011; Tibaduiza, 2013; Yue and Qin, 2001).

Q-statistic (or SPE index). This damage index is based on analyzing the residual data matrix to represent the variability of the data projection within the residual subspace. Denoting $e_{i}$ as the $i$ th row of the matrix $E$, the $Q$-statistic for each experiment can be defined as its squared norm as follows

$$
Q_{i}=e_{i} e_{i}^{\mathrm{T}}=x_{i}\left(\boldsymbol{I}-\boldsymbol{P} \boldsymbol{P}^{\mathrm{T}}\right) x_{i}^{\mathrm{T}}
$$

$T^{2}$-statistic ( $D$ index). The $T^{2}$ index is based on the analysis of the score matrix $\boldsymbol{T}$ to check the variability of the projected data in the new space of the PCs. It can be obtained from the concept of Euclidean distance normalized using the covariance matrix $C_{x}$ as normalization factor. The $T^{2}$-statistic for the $i$ th sample (or experiment) is defined as follows

$$
\boldsymbol{T}_{i}^{2}=\sum_{j=1}^{r} \frac{t_{s i j}^{2}}{\lambda_{j}}=t_{s i} \Lambda^{-1} t_{s i}^{\mathrm{T}}=x_{i} \boldsymbol{P} \Lambda^{-1} \boldsymbol{P}^{\mathrm{T}} x_{i}^{\mathrm{T}}
$$

where $t_{s i}$ is the $i$ th row vector of the matrix $\boldsymbol{T}$, which is the projection of the experiment $x_{i}$ into the new space. Both are related as $t_{s i}=x_{i} \boldsymbol{P}$.

Combined index ( $\Phi$ index). This damage index was previously reported for process monitoring (Alcalá and Qin, 2009). This is a combination of the $Q$ index and $T^{2}$ index, and it was proposed as an alternative for merging information from both into a single value (Yue and Qin, 2001). The following equation mathematically defines this damage index

$$
\begin{aligned}
\phi \text { index } & =Q \text { index }+T^{2} \text { index }=x^{\mathrm{T}} M_{\phi} x \\
& =x^{\mathrm{T}}\left(\boldsymbol{I}-\boldsymbol{P} \boldsymbol{P}^{\mathrm{T}}+\boldsymbol{P} \Lambda^{-1} \boldsymbol{P}^{\mathrm{T}}\right) x
\end{aligned}
$$

I index. This damage index was previously used for clinical studies (Higgins and Thompson, 2002), and it is mainly used in meta-analysis and can be interpreted as a percentage of heterogeneity. In meta-analysis, heterogeneity refers to the variation in study outcomes between studies (Adeyemo and Adediwura, 2012). Equations (7) and (8) define mathematically this damage index

$$
\text { I index }=x^{\mathrm{T}} \boldsymbol{M}_{I} x
$$

where

$$
\boldsymbol{M}_{I}=\left(\begin{array}{ll}
\frac{0}{Q-(k-1)} \\
\frac{(2}{Q} \times 100 \% & \text { for } Q \leq(k-1) \\
\text { for } Q>(k-1)
\end{array}\right.
$$

and $k$ is the number of experiments.

\section{Damage detection methodology}

\section{Problem statement}

One of the most important tasks in SHM corresponds to the damage detection. This is the first step of the process discussed in (Rytter, 1993). In this task, the existence of damage should be determined, and the aim is to know whether there is damage in the structure.

In the literature, several potentially useful techniques for damage detection can be found, and their applicability to a particular situation depends on the size of the critical damages that are admissible in the structure. Almost all of these techniques follow the same general procedure: the structure is excited using actuators and the dynamical response is sensed at different locations throughout the structure. Any damage will change this vibrational response.

The state of the structure is diagnosed by means of processing these data. Several studies have shown that the detection of changes in a structure depends on the distance from the damage to the actuator as well as the configuration of the sensor network. This article is concerned with the practical application of a methodology for the problem of detection of damages in structures by using statistical data-driven models built from structural dynamic responses when the structure is known to be healthy. In the following sections, a detailed description of the methodology is presented. The methodology is the basis of this work, and it can be adapted and extended to localize and classify damages.

\section{Overview}

The damage detection methodology proposed in this article involves the use of a multiactuator piezoelectric system (distributed piezoelectric active network), PCA, and some damage indices. In general terms, the 


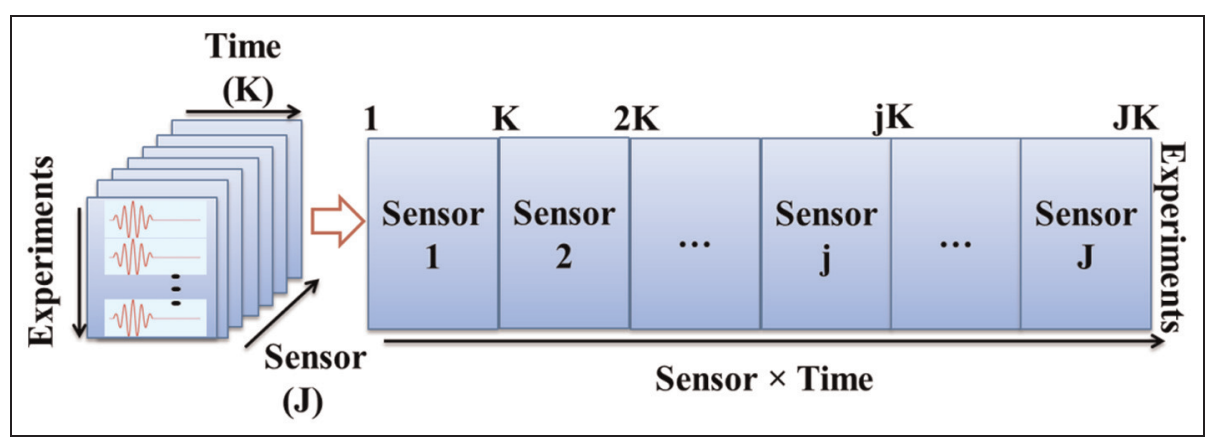

Figure I. Decomposition of data collected from experiments to two-dimensional $(I \times J K)$.

practical application of this approach is performed in two stages:

(1) Baseline modeling: In this stage, experiments of the structure are performed when it is well known that the structure is undamaged. Data gathered by sensors are organized and preprocessed to obtain the statistical data-driven baseline models based on PCA. Scores and indices obtained in this stage are also stored as the baseline indicators.

(2) Data projections onto the models: In this stage, the structure to be studied is subject to the same experiments that are performed on the healthy one. Data are organized, pre-processed, and projected onto the models obtained in the previous stage. Consequently, scores and indices are calculated and compared with the obtained ones using the healthy structure.

Details about the multiactuator piezoelectric system and the way of performing the experiments are presented in the next sections. Although the theoretical background about PCA was introduced in the previous section, the procedure for (1) organizing the data gathered by experiments, (2) building the baseline PCA model, and (3) projecting the data from new experiments are described also in the following sections.

\section{Experimental setup and data acquisition}

The structure to be tested is instrumented with several lead zirconate titanate (PZT) transducers bounded on the surface. It is isolated in order to remove environmental noise and the boundary conditions. To perform the excitation and to collect the vibrational responses from the structure, several actuation phases are used. In every actuation phase, a single PZT transducer is used as actuator and the others as sensors that receive the wave propagated across the structure at different points. Typically, a BURST signal with a frequency defined for each structure is used as signal excitation.
This frequency is chosen once a sweep frequency response test is performed. In some structures, depending on its complexity, this signal should be amplified by using a wideband power amplifier. To remove the noise in the signals, one experiment consists of repetitions which are averaged. Several structural states are studied for each structure. In general, these states contain the healthy structure and different damages.

\section{Preprocessing}

The signals collected from the experiments are stored by the acquisition system in a matrix with dimensions $(I \times K)$, where $I$ represents the number of experiments and $K$ the number of samples or time. At the same time, because there are $J$ matrices with the information from each sensor by each actuation phase, a threedimensional (3D) matrix by each actuation phase is obtained, which means one matrix by each PZT. In order to organize the information, a bi-dimensional matrix is built, where data from each sensor are located beside the other sensors as shown in Figure 1.

As a preliminary step to implement the PCA methodology, a pre-processing of the data collected in each actuation phase is performed. For this kind of datasets (unfolded matrix), the authors found that group-scaling presents the best results because it considers changes between sensors and does not process them independently (Tibaduiza et al., 2012). More details about how to apply group-scaling can be found in Tibaduiza et al. (2012). Once the normalization is applied, the mean trajectories (by sensor) are removed and all sensors are made to have equal variance. As a consequence, the experimental trajectories of the sensors and their standard deviations, often nonlinear in nature, are removed from the data.

\section{Baseline model building and calculation of damage indices using PCA}

In the first stage, a PCA baseline model is built for each actuator phase (PZT1 as actuator, PZT2 as actuator, 


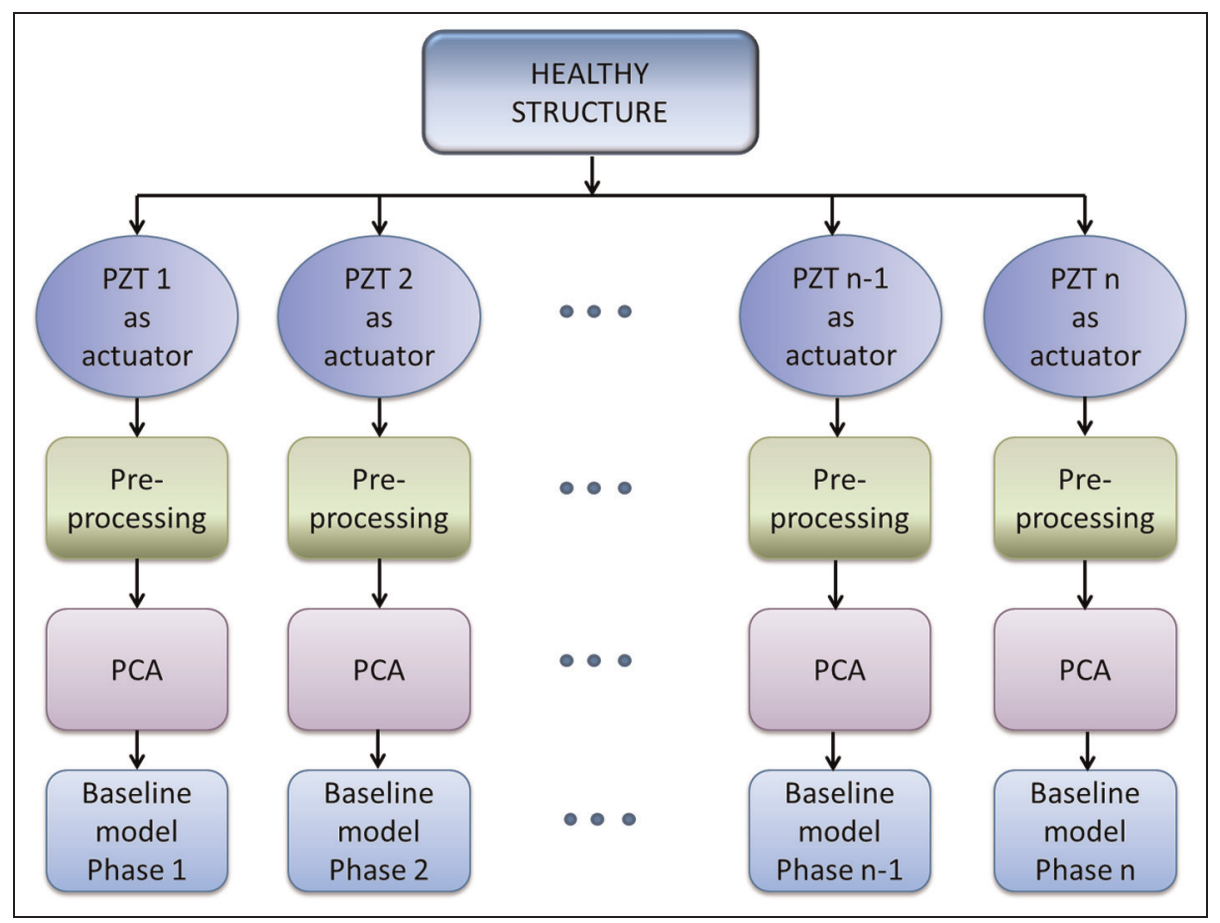

Figure 2. Baseline definition methodology.

etc.) using the signals recorded by sensors during the experiments with the undamaged structure. The scheme showed in Figure 2 summarizes the procedure. PCA baseline modeling essentially consists of calculating the matrix $\boldsymbol{P}$ for each phase as was explained in the "Theoretical background" section.

In the second stage, experiments are performed using the structure in different possible states or scenarios (undamaged and different damages). Figure 3 illustrates the procedure. These signals are pre-processed and organized according to the PCA modeling procedure. Afterward, they are projected on the corresponding PCA model $(\boldsymbol{T}=\boldsymbol{X} \boldsymbol{P})$. A selected number of the first PCs (scores $\boldsymbol{T}$ ) are obtained. In addition, some damage indices are calculated by each baseline PCA model using equations (4) to (7) introduced in the "Theoretical background" section.

The idea behind the use of different damage indices is to determine from different points of view the presence of damages in the structure, which means to try to remove the possibility of producing false alarms as a result of a bad analysis from one of these indices. From this point of view, each damage index allows comparing the new data in the model from different points of view as it was explained in the "Theoretical background" section. Figure 4 presents a diagram of the overall damage detection methodology.

\section{Experimental setup}

The validation of the damage detection methodology is carried out by using data from experiments performed on two different specimens: an aircraft turbine blade and an aircraft skin panel. Figure 5 shows the first structure; an important feature to highlight in this structure is its irregular form and the presence of a stringer in both faces. This blade was instrumented with seven piezoelectric transducers attached on the surface: three of them were distributed in one face and the others on the other face.

The second structure is an aircraft skin panel. This structure is divided in small sections by means of stringers and ribs as shown in Figure 6. Two of these sections were instrumented with six PZT transducers - two in upper section, two in lower section, and two in the stringer (Figure 7).

As excitation signal, a BURST signal is applied. To determine the carrier central frequency for the actuation signal in each structure, a frequency sweep was performed and spectral analysis of each signal was explored. As a result of this analysis, a BURST signal with $250 \mathrm{kHz}$ and three peaks was defined for the aircraft turbine blade and a signal with $205 \mathrm{kHz}$ and nine peaks for the aircraft skin panel.

Before applying the signal to the structure, it is amplified to $50 \mathrm{~V}$ using a wideband power amplifier. The collection of data is performed in different phases; in each phase, one PZT is selected as actuator and the excitation signal is applied, then the vibrational response is collected by the other PZT transducers attached to the structure in different positions.

In the aircraft turbine blade case, 10 different states of the structure were studied, in which the first one corresponds to the healthy structure and the others are 


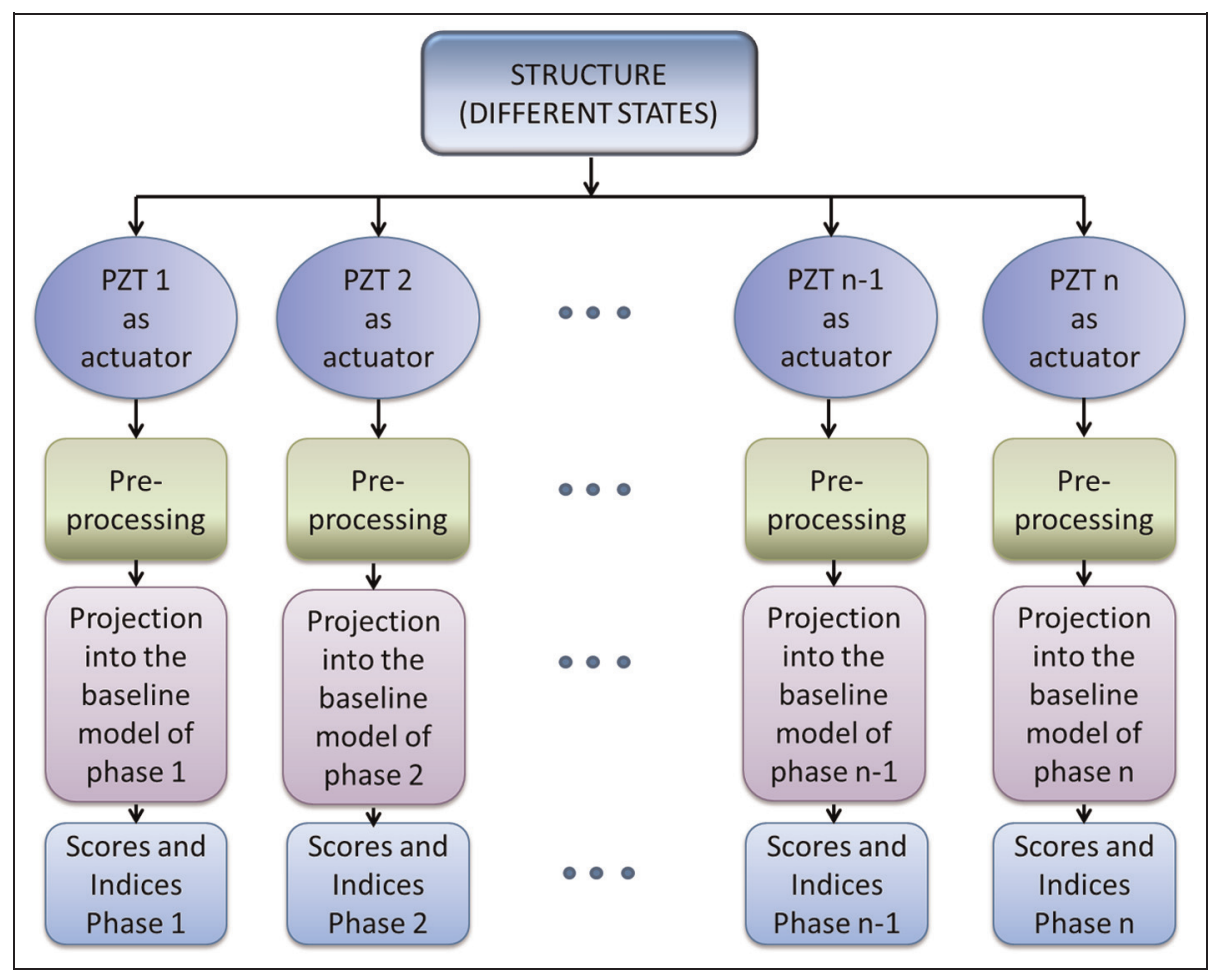

Figure 3. Data projection into the PCA models.

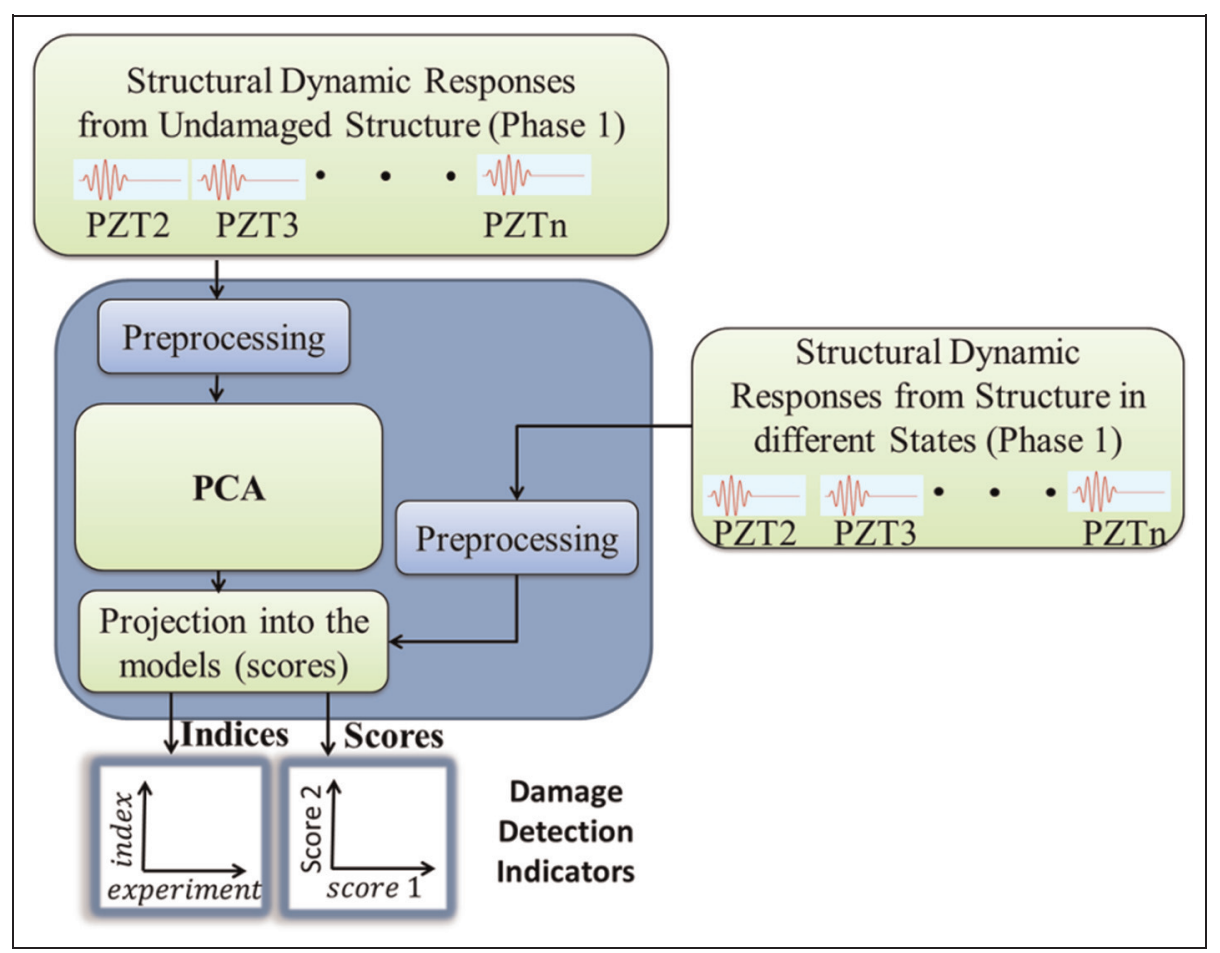

Figure 4. Damage detection methodology.

nine damages which are structural modifications by adding a mass at different positions of the structure as in Figure 8. A total of 140 experiments were performed and recorded (50 with the undamaged structure and 10 per damage). To build the PCA models, $80 \%$ of the whole dataset collected using the undamaged structure 


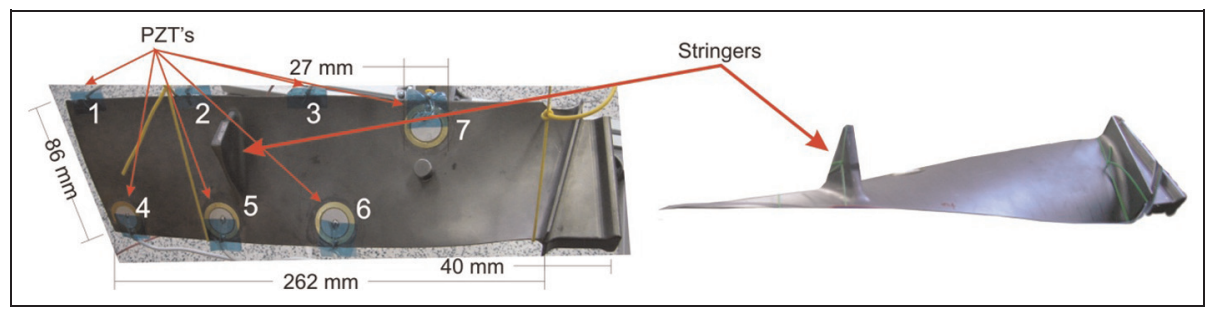

Figure 5. Aircraft turbine blade.

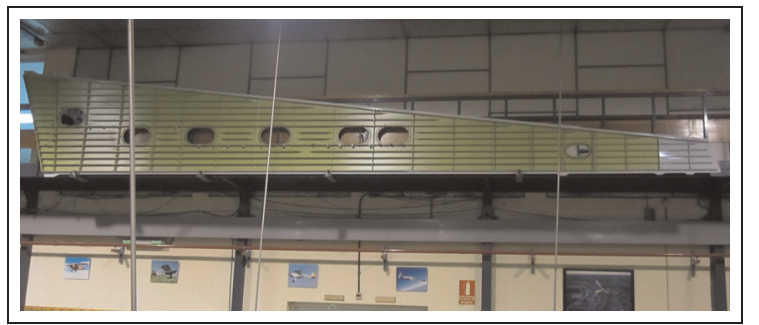

Figure 6. Aircraft skin panel.

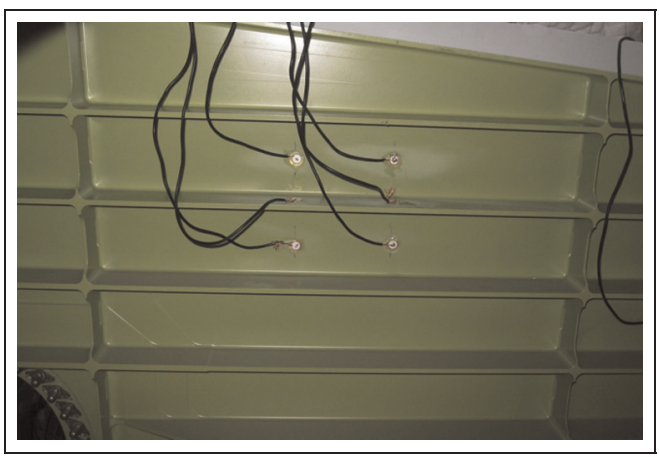

Figure 7. Sections tested with the PZT location.

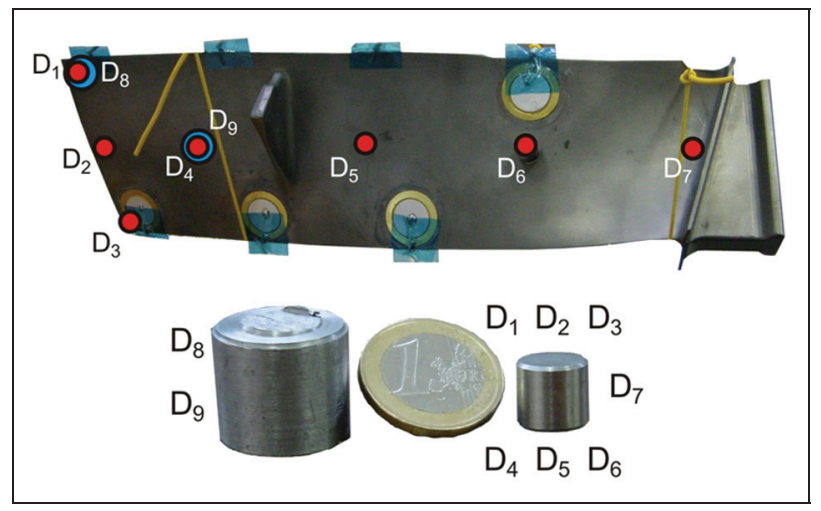

Figure 8. Damage distribution on the aircraft turbine blade.

was used. Signals from other $20 \%$ and the whole dataset of the damaged structure were used for testing the approaches.
In the second specimen, three different damages were tested, where each damage corresponds to structural changes by adding a mass in a specific location (Figure 9). In total, four different states of the structure were analyzed: the healthy structure and three damages; 450 experiments were performed and recorded: 150 with the undamaged structure and 100 per damage. To ensure a good signal-to-noise ratio, each signal was averaged 10 times.

\section{Experimental results}

The validation of the damage detection methodology is carried out by using data from experiments performed on an aircraft turbine blade and an aircraft skin panel. As mentioned in the previous section where the structures are described, the aircraft turbine blade was instrumented with seven PZT transducers, therefore seven actuator phases were accomplished. Besides, nine defects were simulated. On the other hand, the aircraft skin panel was instrumented with six PZT transducers (six actuator phases) and three defects were simulated.

\section{Distribution of variance}

Once the baseline PCA models were built by using experiments from the healthy structure, an analysis of the variance captured by each PC was achieved. This analysis is important in order to ensure that enough variance is retained into the model, which allows performing an optimal reduction. The distribution of the retained variance in four of the actuator phases of each structure is depicted in Figures 10 and 11. Only the components with a significant variance value are shown. The components with the highest variance represent the most important pattern in the data with the largest quantity of information. Figure 10 shows that using the aircraft turbine blade, the first two components represent more than $80 \%$ of the cumulative variance in each model. Similar results are obtained in other phases.

On the other hand, from Figure 11, it can be seen that the percentage of the variance of each component in the aircraft skin panel is lesser than that obtained in the previous structure. 


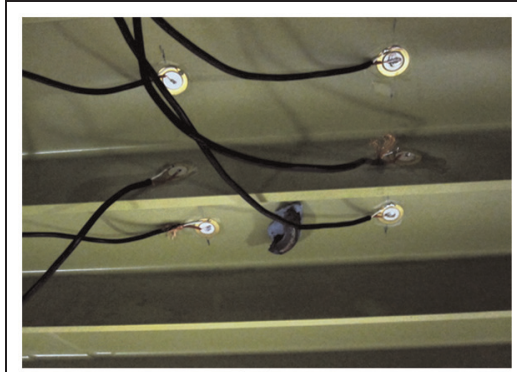

(a)

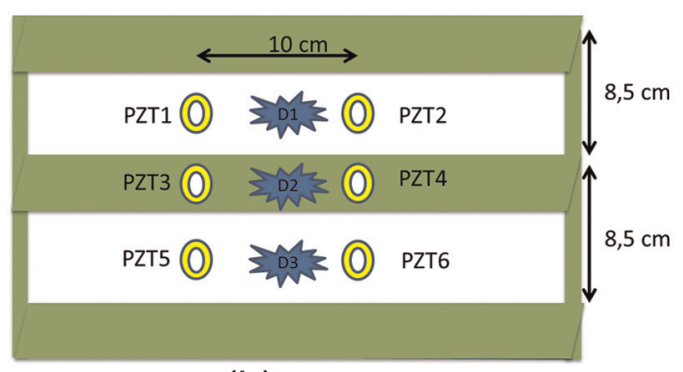

(b)

Figure 9. (a) Damage description and (b) positions of the damages in the sections of the aircraft skin panel.
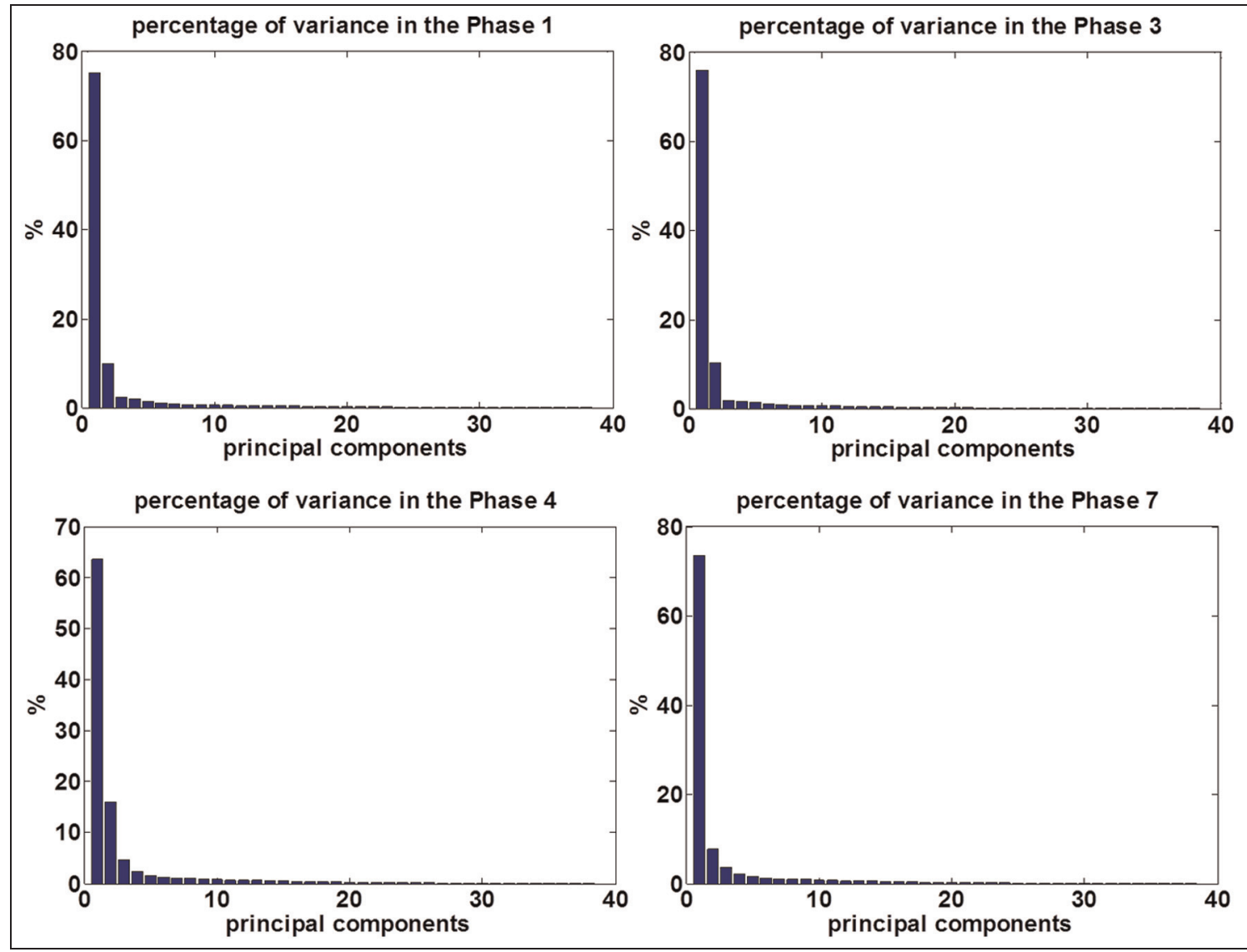

Figure 10. Distribution of the variance in phases $1,3,4$, and 7 in the aircraft turbine blade.

Although the first two components are the most significant, these only contain $37 \%$ of the cumulative variance. This difference can be explained by the differences between the structures.

In order to define standard criteria for the comparison and for maintaining simplicity in the analysis and the visualization tasks, only two scores are used in both cases in order to show how this choice affects the results of each structure for each of the proposed approaches.

A previous work showed the advantages and disadvantages in the use of the score plots for detecting damages (Mujica et al., 2011). This work aims to show how the results in these plots can be different in some cases, thus requiring additional analysis tools such as damage indices for performing a good detection and identification task.

\section{PCA score plots}

The projections of each experiment onto the PCs subspace are called scores. Depicting two scores in a scatter plot allows visualizing the structure of the original data; it is known as score plots. 


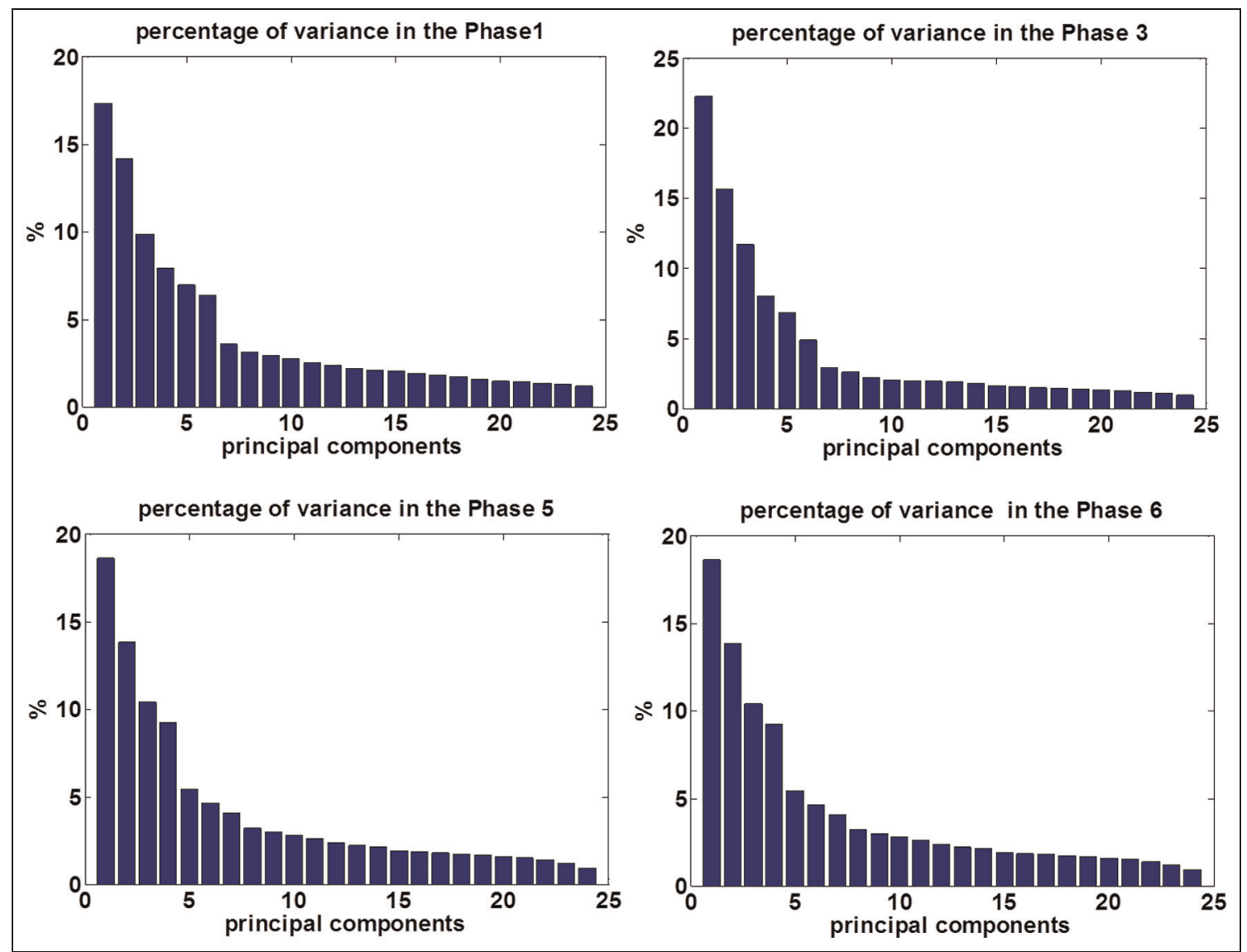

Figure I I. Distribution of the variance in phases I, 3, 5, and 6 in the aircraft skin panel.

Figures 12 and 13 show the score plots of the first and second PCs for different actuation phases of the aircraft turbine blade and aircraft skin panel, respectively. As was previously mentioned, each actuation phase corresponds to the experiments performed when just one PZT transducer is defined as actuator and the remaining are used as sensors.

To validate the results and considering that the state or condition of the specimen (undamaged, damage 1 , damage 2 , etc.) is a priori known in each experiment, each projection is labeled; in this way, each group of data can be identified. Different shapes and colors represent the different conditions of the specimens.

As can be seen from Figure 12, corresponding to the aircraft turbine blade, all the damages can be clearly distinguished from the undamaged structure state (plus sign); similar results are obtained in the other phases which are not showed. This separation can be used to confirm changes in the structure, and it can be defined as an abnormal situation. This means that it is possible to detect the presence of damages. Besides, it is possible to distinguish and separate some datasets between them, even being very close, which means that the vibrational responses are similar.
On the other hand, from Figure 12, it can be seen that in the aircraft skin panel, it is also possible to identify the dataset gathered from the damaged structure. However, in contrast to the turbine blade, this separation is not clear in all phases (phases 1 and 3). In this context, it is appropriate to admit that this result is related to the complexity of the structure. This result implies that it is necessary to use another type of measurement or statistic to obtain a better discrimination of the presence of damage in any structure for each one of the phases. In this work, the use of the damage detection plots by means of the use of different indices is proposed as a solution to this problem. The following subsections show the results obtained by using the different indices explained in the "Theoretical background" section.

\section{Damage index plots}

As was mentioned in the "Theoretical background" section, there exist in the literature several statistical measurements that can explain the behavior of the projected data into the model. If the original data and the baseline data differ, it should be reflected in the scores and/or these indices. 


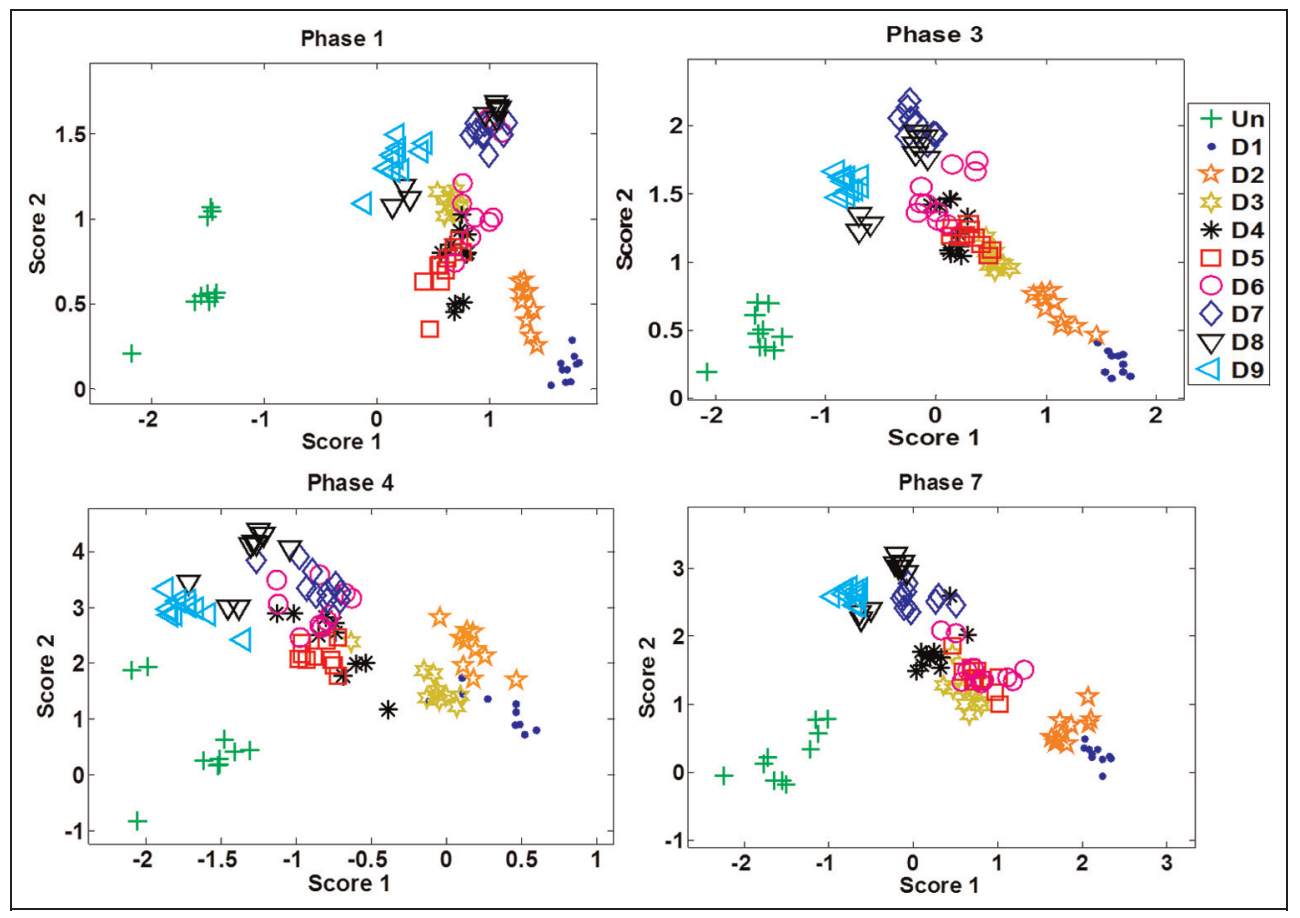

Figure I2. Score I versus score 2 in the aircraft turbine blade in the phases I, 3, 4, and 7.

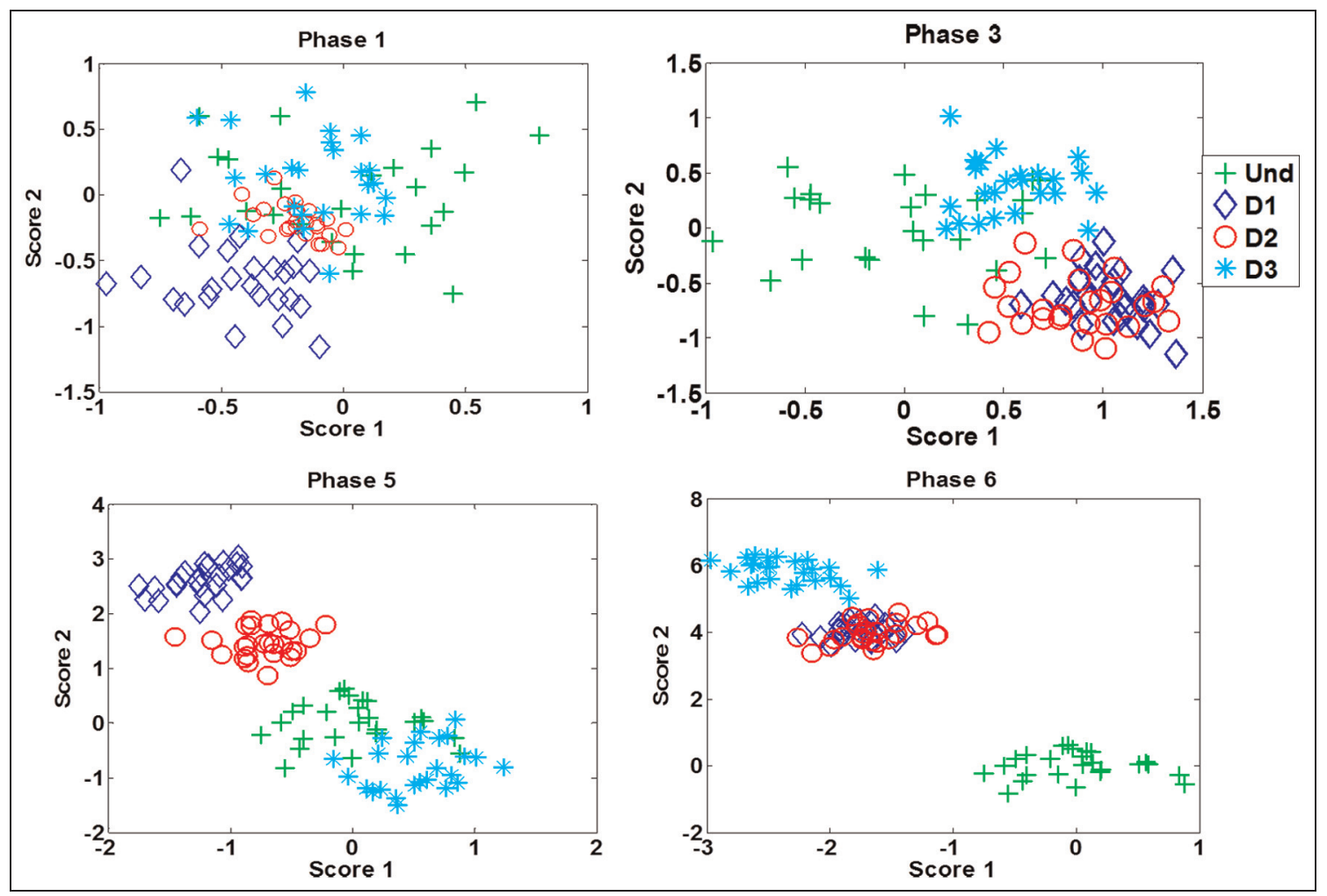

Figure I3. Score I versus score 2 in the aircraft skin panel in the phases I, 3, 5, and 6.

$T^{2}$ index. From Figure 14, the $T^{2}$ index by each experiment using the aircraft turbine blade in four actuator phases can be seen. The results show that each actuator phase depicts different ways of visualizing all the datasets with the information of the damages. In all these actuation phases, damages 1,2, and 3 have values of $T^{2}$ 


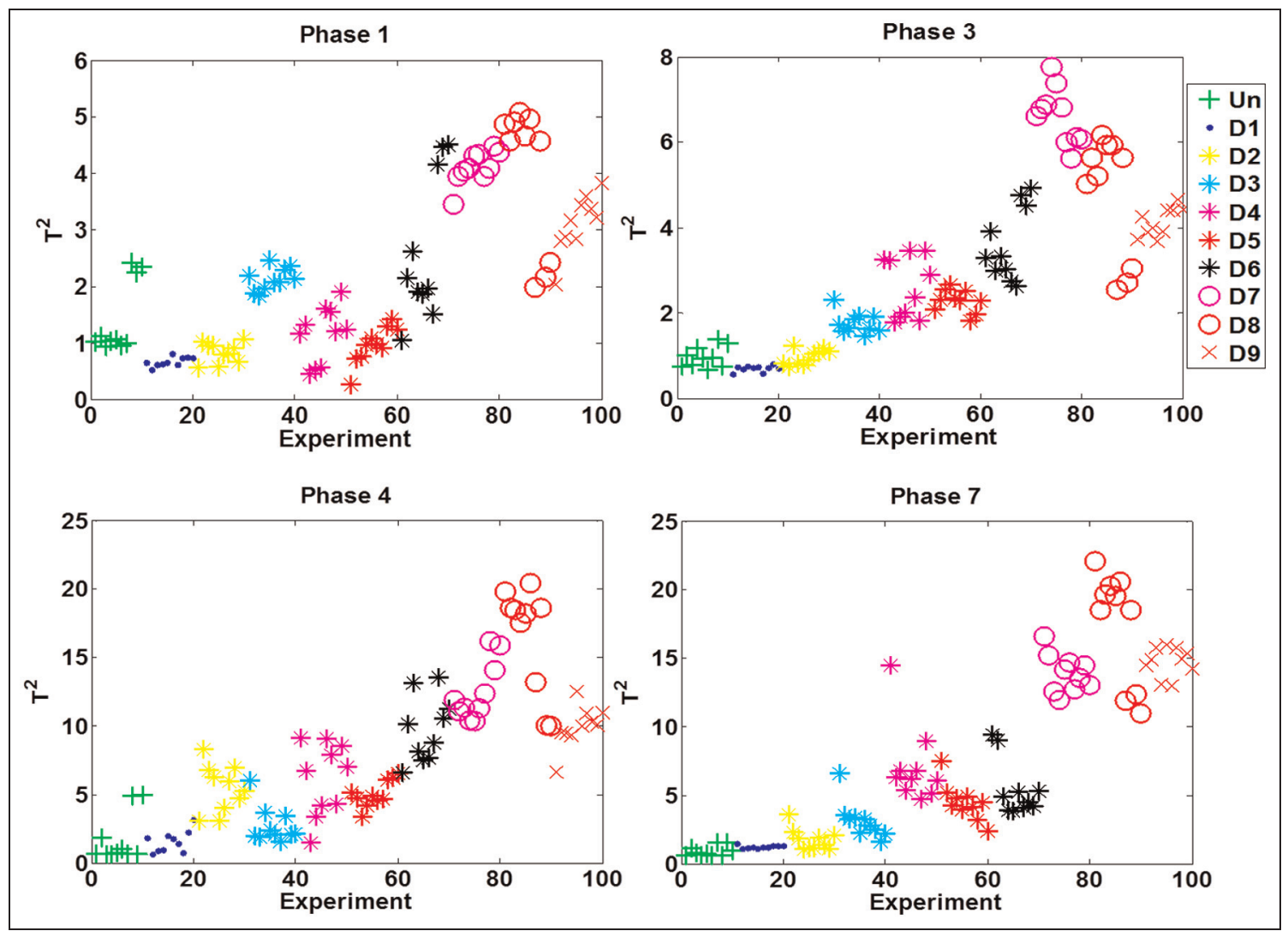

Figure 14. $T^{2}$ index in the aircraft turbine blade.

index similar to the undamaged state, and therefore, they cannot be considered as damages. Besides, from the evaluation of phases 1 and 4 , it can be seen that damage 4 has values similar to the undamaged state. In contrast, a higher separation is found in phases 3 and 7. The remaining damages are well separated from the undamaged state in all the actuation phases.

Considering the aircraft skin panel, Figure 15 shows the plots of the $T^{2}$ index by each experiment. As it is disclosed, damage 1 is clearly separated from the undamaged state in all the phases. Additionally, all the damages are well identified by phases 1 and 3 . On the contrary, phases 5 and 6 show that damages 2 and 3 have value similar to $T^{2}$ index in the undamaged state.

$Q$ index. From Figure 16, the $Q$ index calculated by each experiment using the aircraft turbine blade in the actuator phases 1, 3, 4, and 7 can be seen. In difference with the $T^{2}$ index, phase 1 allows identifying damage 1 . Additionally, in all the cases, damage 3 is well separated from the undamaged state. The remaining damages are not well identified.

Figure 17 shows the results of the $Q$ index which is calculated using the data from the aircraft skin panel. The results in this structure show that in all the phases, the three damages are clearly separated from the undamaged state and, additionally, these states are easily distinguishable between them.

$I^{2}$ index. From Figure 18, it can be seen that the $I^{2}$ index allows identifying damage 1 by means of the actuation phases 1 and 4 in the aircraft turbine blade. Additionally, some experiments of damages 3, 4, 5, and 8 allow defining these states as damages. One important feature to highlight in comparison with the $Q$ index is that in this plot, only significant differences are visible. In this way, values near to the healthy state are defined as zero.

Figure 19 shows the results in the aircraft skin panel with the $I^{2}$ index by each experiment. Similar to the results obtained with the $Q$ index, all the damages are clearly separated from the undamaged state. This result confirms the big differences between each state and the data from the healthy structure.

Combined index ( $\phi$ index). Figure 20 shows the results of the $\phi$ index in the aircraft turbine blade in four actuation phases. These results show how damage 3 is clearly identified in every actuation phase. Additionally, the remaining damages need to be identified by the analysis of the different phases. Similar results are obtained in the other actuation phases. 


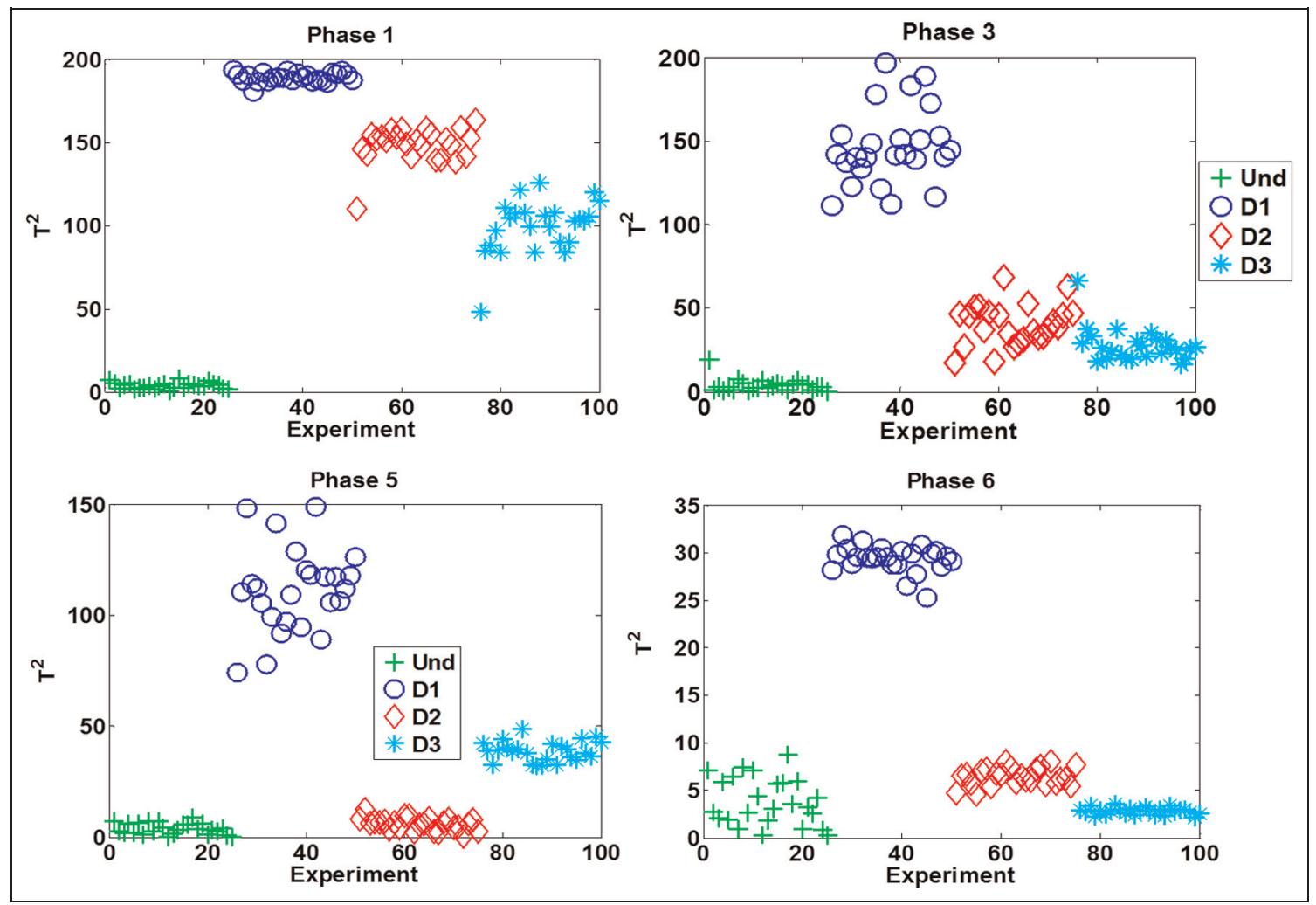

Figure I5. $T^{2}$ index in the aircraft skin panel.
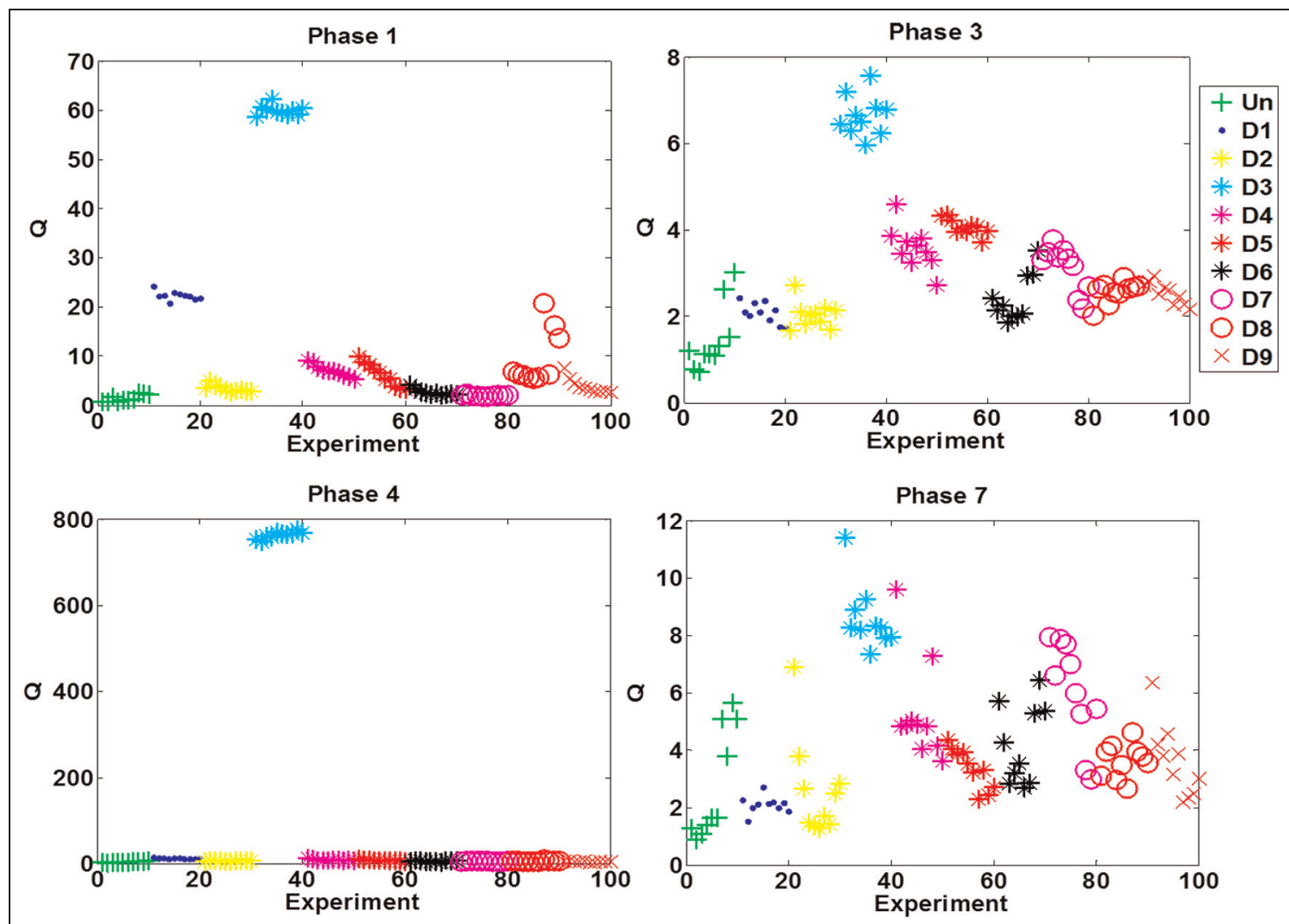

Figure 16. $Q$ index in the aircraft turbine blade. 


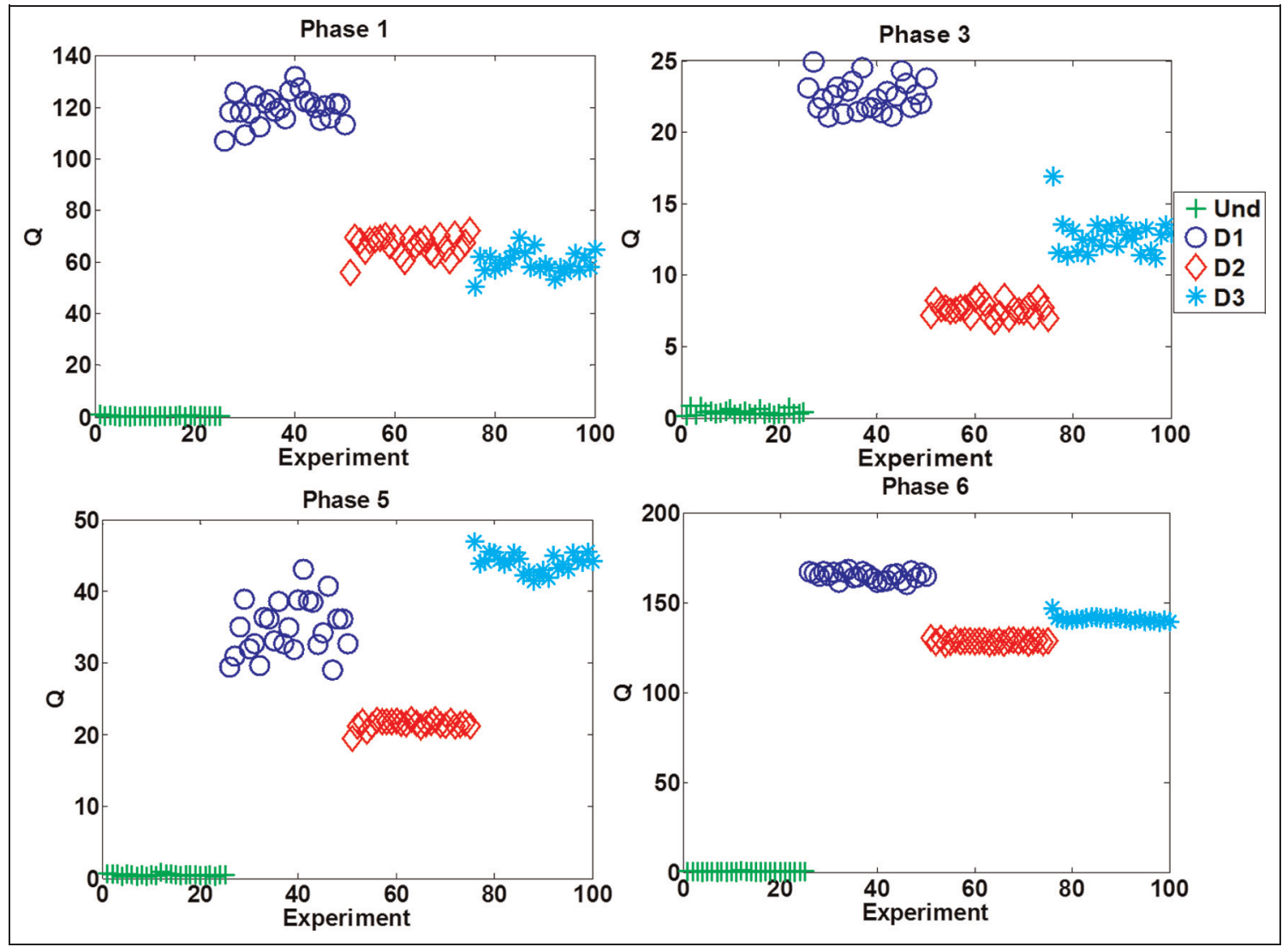

Figure 17. $Q$ index in the aircraft skin panel.

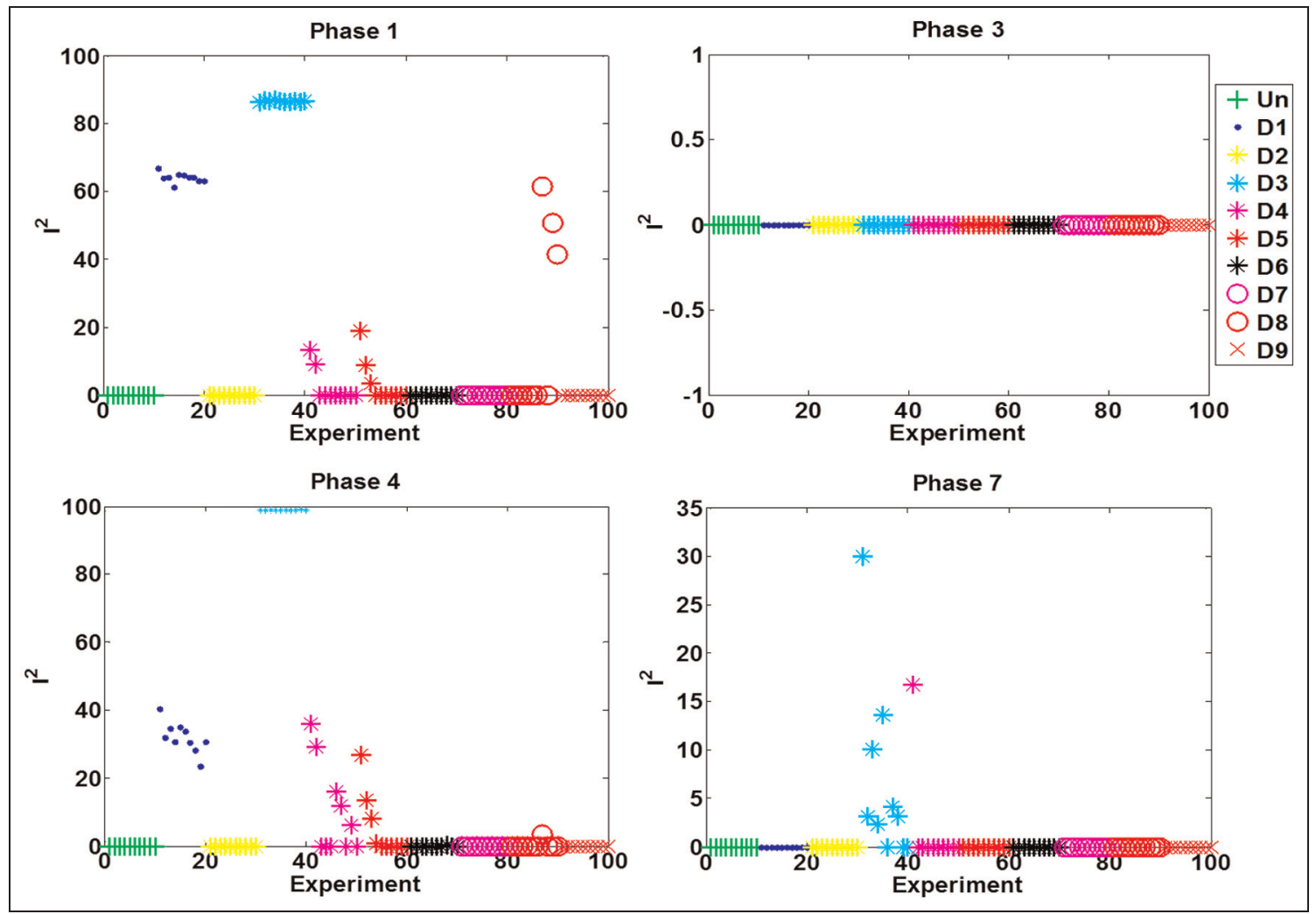

Figure 18. $I^{2}$ index in the aircraft turbine blade. 


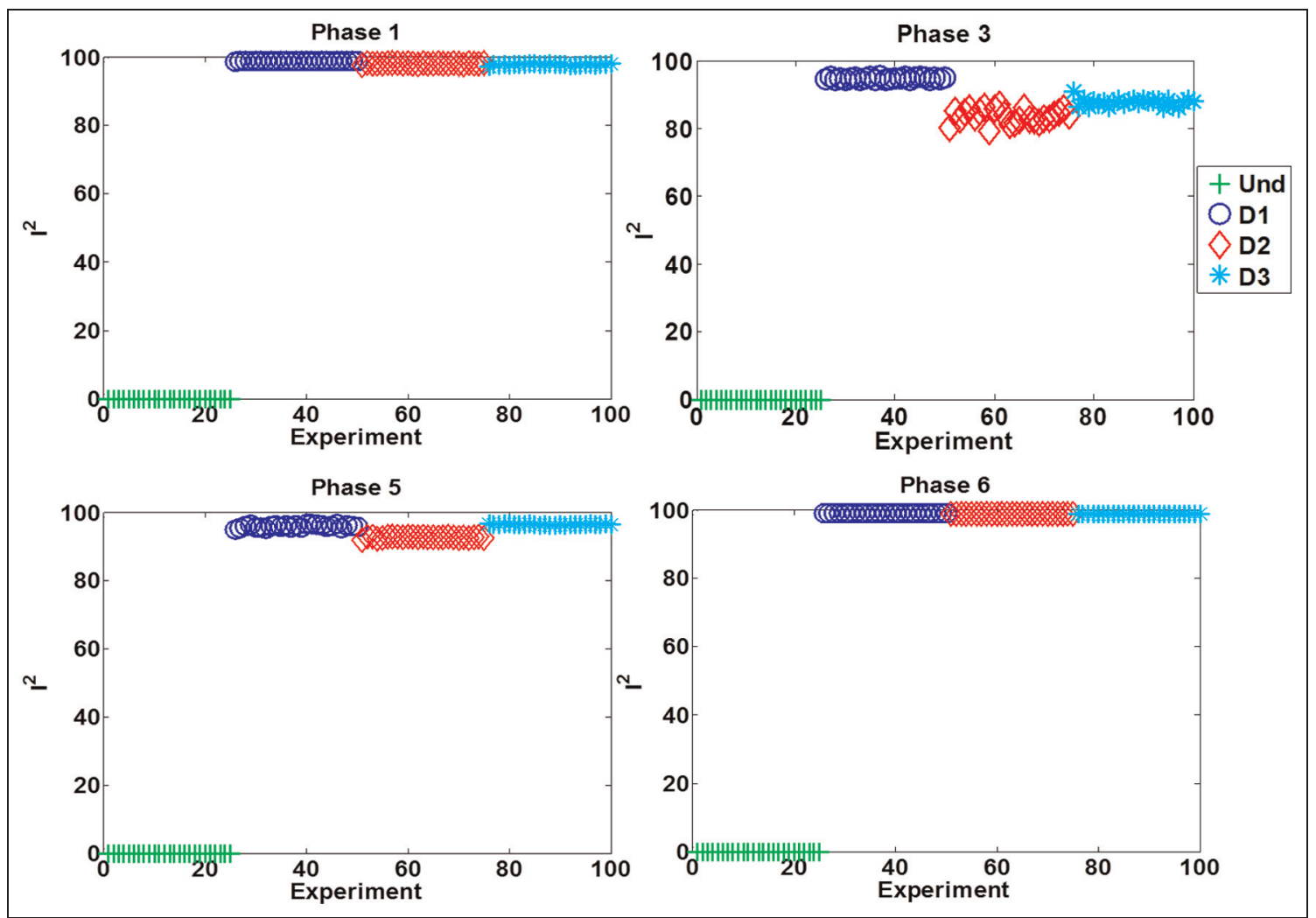

Figure 19. $I^{2}$ index in the aircraft skin panel.
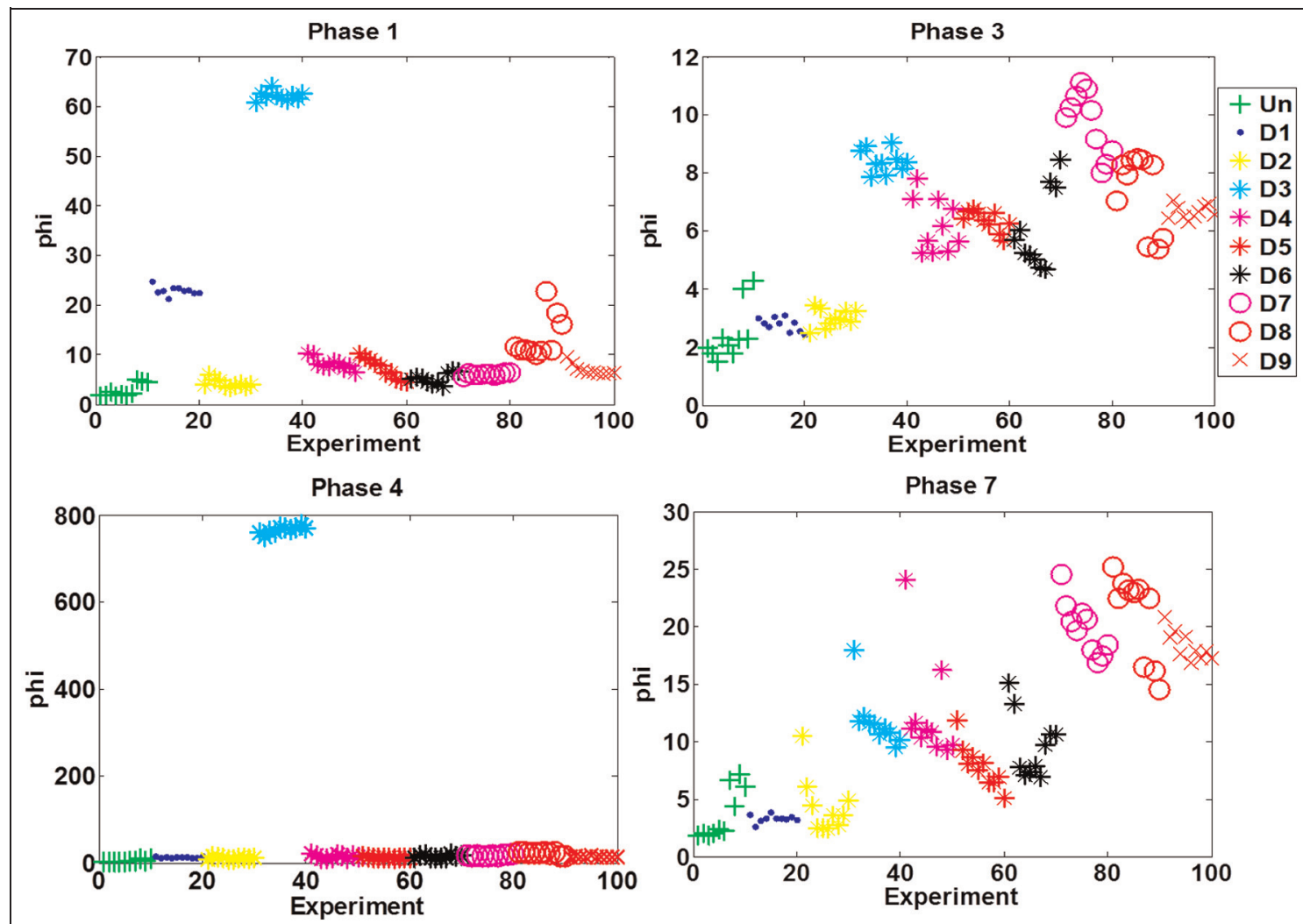

Figure 20. Combined index in the aircraft turbine blade. 


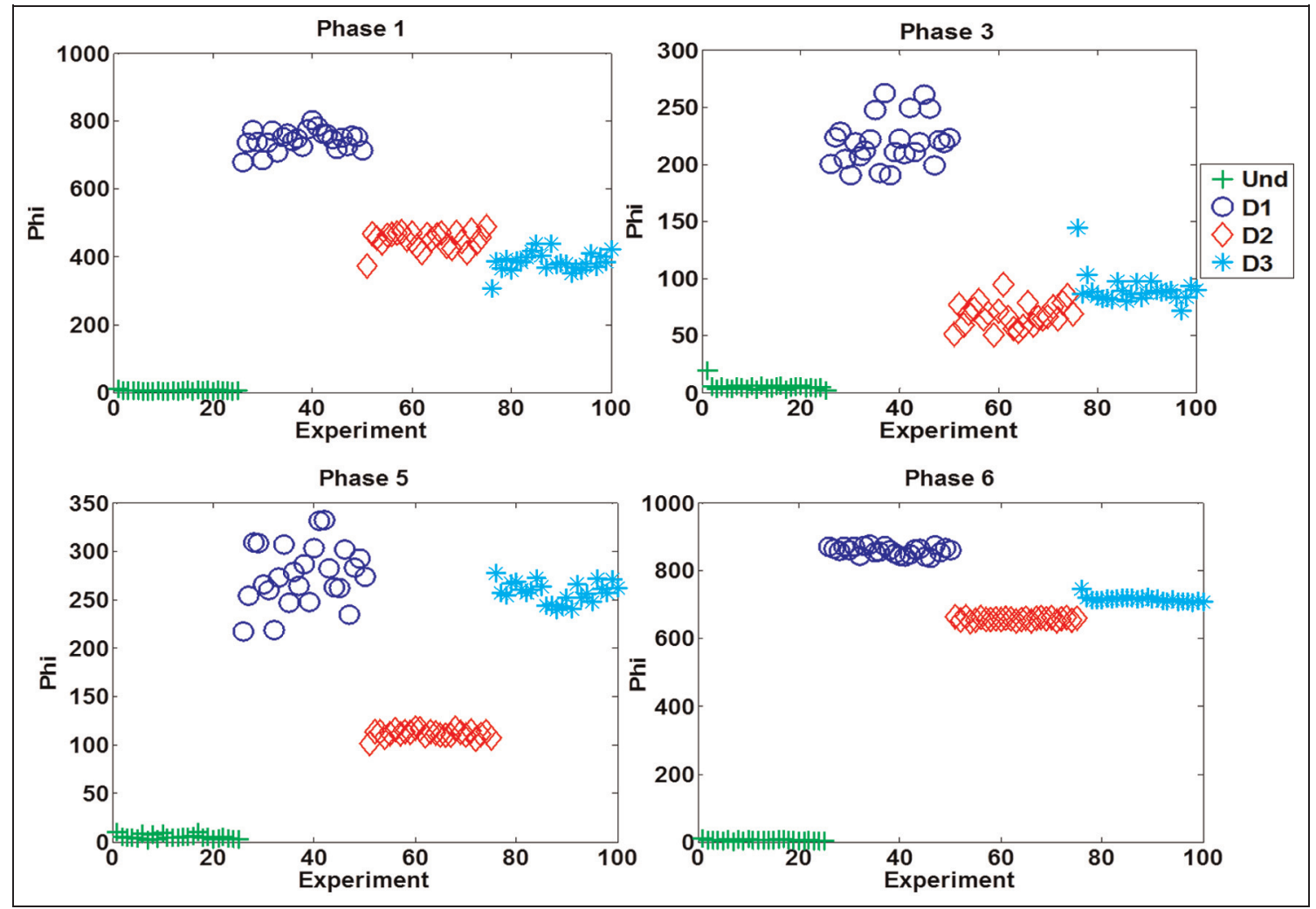

Figure 21. Combined index in the aircraft skin panel.

Finally, Figure 21 shows the results of the application of this index to the aircraft skin panel. In this case, in the same manner as with the $Q$ and $I^{2}$ indices, all the damages are clearly separated from the undamaged state.

\section{Conclusion}

The proposed methodology is a data-based approach where sensor data fusion, feature extraction, and pattern recognition are evaluated by using different damage indices. A first major advantage of the methodology is that the need to develop and validate a numerical model is obviated. Additionally, and in contrast to standard Lamb waves-based methods, there is no necessity of directly analyzing the complex timedomain traces containing overlapping, multi-modal, and frequency dispersive wave propagation which distorts the signals and makes their analysis difficult. However, within the proposed methodology, it is not possible to provide a multi-damage detection which is able to identify several occurring damages independently.

The performance of the methodologies presented for damage detection using PCA as pattern recognition tool and four damage indices $\left(T^{2}, Q, \Phi\right.$, and $\left.I^{2}\right)$ has been tested using an aircraft skin panel and an aircraft turbine blade. The results have revealed that the approaches have potential for real applications and they can be used in a combined way to evaluate the state of a structure. The results also showed that there are differences between the data from the undamaged structure and the different damages; these differences can be used to define the presence of damages in the structure. Similarly, the graphs presented allowed, in most cases, separating and distinguishing the damages between them.

Comparing the results from the two specimens, it was shown that the score plots are not very useful when the variance contained in the scores to use is not a significant value; in these cases, the use of a combined analysis with the damage indices plots can be used for detecting and classifying damages with better results. In addition, it is also possible to distinguish some datasets which can be used for damage classification in a further analysis using, for instance, some additional pattern recognition technique.

In general, it is possible to conclude that, in all the cases, the evaluation of all the phases allows defining the presence of a damage in the structure. This full analysis is a necessary step because as it was shown in the results, each phase defines the states in a different manner. In the case when a high number of actuation steps need to be evaluated to determine the presence of a damage, the methodology requires the use of data fusion to provide a unique result and reduce the review 
of multiple plots; by this reason, it is suggested for future work to use a method to combine all the results and produce a simplified plot

To determine which damage index presents best results in the damage detection process, a study of the influence of environmental and operational conditions on the damage detection process needs to be performed.

\section{Acknowledgements}

The authors would like to thank the support from the "Agència de Gestió d'Ajuts Universitaris i de Recerca" of the "Generalitat de Catalunya," "Escola Universitària d'Enginyeria Tècnica Industrial de Barcelona (EUETIB)," "Universitat Politécnica de Catalunya," and "Universidad Politécnica de Madrid."

\section{Declaration of conflicting interests}

The authors declared no potential conflicts of interest with respect to the research, authorship, and/or publication of this article.

\section{Funding}

This work has been supported by the "Ministerio de Ciencia e Innovación" in Spain through the coordinated research projects DPI2008-06564-C02-01/02 and DPI2011-28033-C03-01.

\section{References}

Adeyemo E and Adediwura A (2012) Assessing heterogeneity of effect size on sample size in a meta-analysis of validity studies. Mediterranean Journal of Social Sciences 3(1): 219-227.

Alcalá C and Qin SJ (2009) Unified analysis of diagnosis methods for process monitoring. In: 7th IFAC symposium on fault detection, supervision and safety of technical processes, Barcelona, 30 June-3 July.

Farrar CR, Sohn H and Park G (2004) A statistical pattern recognition paradigm for structural health monitoring. In: 9th ASCE specialty conference on probabilistic mechanics and structural reliability, Albuquerque, NM, 26-28 July.

Higgins JPT and Thompson SG (2002) Quantifying heterogeneity in a meta-analysis. Statistics in Medicine 21: $1539-1558$.
Jolliffe IT (2002) Principal Component Analysis. New York: Springer.

Kerschen G, De Boe P, Golinval JC, et al. (2005) Sensor validation using principal component analysis. Smart Materials and Structures 14: 36-42.

Manson G (2002) Identifying damage sensitive, environment insensitive features for damage detection. In: $3 \mathrm{rd}$ international conference on identification in engineering systems, Swansea University, Swansea, 15-17 April.

Manson G, Worden K, Holford K, et al. (2001) Visualization and dimension reduction of acoustic emission data for damage detection. Journal of Intelligent Material Systems and Structures 12: 529-536.

Mujica LE, Rodellar J, Fernández A, et al. (2011) Q-statistic and $\mathrm{T}^{2}$-statistic PCA-based measures for damage assessment in structures. Structural Health Monitoring: An international Journal 10(5): 539-553.

Mujica LE, Vehí J, Ruiz M, et al. (2008) Multivariate statistics process control for dimensionality reduction in structural assessment. Mechanical Systems and Signal Processing 22: 155-171.

Rytter A (1993) Vibration based inspection of civil engineering structures. PhD Thesis, Department of Building Technology and Structural Engineering, Aalborg University, Aalborg.

Tibaduiza DA (2013) Design and validation of a structural health monitoring for aeronautical structures. $\mathrm{PhD}$ Thesis, Universitat Politècnica de Catalunya, Barcelona.

Tibaduiza DA, Mujica LE and Rodellar J (2011) Comparison of several methods for damage localization using indices and contributions based on PCA. Journal of Physics: Conference Series 305: 012013.

Tibaduiza DA, Mujica LE and Rodellar J (2012) Damage classification in structural health monitoring using principal component analysis and self organizing maps. Structural Control and Health Monitoring 20: 1303-1316.

Tibaduiza DA, Torres MA, Mujica LE, et al. (2013) A study of two unsupervised data driven statistical methodologies for detecting and classifying damages in structural health monitoring. Mechanical Systems and Signal Processing 41(1-2): 467-484.

Yue H and Qin SJ (2001) Reconstruction-based fault identification using a combined index. Industrial \& Engineering Chemistry Research 40: 4403-4414. 